\title{
Electrical Stimulation of Semicircular Canal Afferents Affects the Perception of Head Orientation
}

\author{
Richard F. Lewis, ${ }^{1,2,3}$ Csilla Haburcakova, ${ }^{1}$ Wangsong Gong, ${ }^{1}$ Daniel Lee, ${ }^{2}$ and Daniel Merfeld ${ }^{1,2}$ \\ ${ }^{1}$ Jenks Vestibular Physiology Laboratory, Massachusetts Eye and Ear Infirmary, and Departments of ${ }^{2}$ Otology and Laryngology, and ${ }^{3}$ Neurology, Harvard \\ Medical School, Boston, Massachusetts 02114
}

Patients with vestibular dysfunction have visual, perceptual, and postural deficits. While there is considerable evidence that a semicircular canal prosthesis that senses angular head velocity and stimulates canal ampullary nerves can improve vision by augmenting the vestibulo-ocular reflex, no information is available regarding the potential utility of a canal prosthesis to improve perceptual deficits. In this study, we investigated the possibility that electrical stimulation of canal afferents could be used to modify percepts of head orientation. Two rhesus monkeys were trained to align a light bar parallel to gravity, and were tested in the presence and absence of electrical stimulation provided by an electrode implanted in the right posterior canal. While the monkeys aligned the light bar close to the true earth-vertical without stimulation, when the right posterior canal was stimulated their responses deviated toward their left ear, consistent with a misperception of head tilt toward the right. The deviation of the light bar from the earth-vertical exceeded the torsional deviation of the eyes, indicating that the perceptual changes were not simply visual in origin. Eye movements recorded during electrical stimulation in the dark were consistent with isolated activation of right posterior canal afferents, with no evidence of otolith stimulation. These results demonstrate that electrical stimulation of canal afferents affects the perception of head orientation, and therefore suggest that motionmodulated stimulation of canal afferents by a vestibular prosthesis could potentially improve vestibular percepts in patients lacking normal vestibular function.

\section{Introduction}

The vestibular labyrinth senses angular and linear head motion and head orientation relative to gravity, and this information contributes to percepts of head orientation and motion (Merfeld, 2012), postural control (Peterka, 2002), and the vestibulo-ocular reflex (VOR), which stabilizes images on the retina (Paige, 1983). When vestibular end-organs are damaged, these normal processes are impaired. Patients with vestibular deficits therefore experience abnormal percepts of orientation and motion (Lewis, 1996), imbalance (Diener and Dichgans, 1988), and impaired vision (Minor, 1998). Since vestibular damage and the resultant symptoms are often poorly responsive to available therapies, development of vestibular prostheses has become an area of considerable interest (for review, see Merfeld and Lewis, 2012).

The semicircular canals transduce angular head velocity, and since the polarities of all hair cells in each canal crista are aligned, the activity in each canal afferent modulates in a qualitatively similar manner during head rotation. Direct electrical stimulation of canal ampullary nerves could therefore encode head an-

\footnotetext{
Received Jan. 9, 2013; revised April 24, 2013; accepted April 28, 2013.

Author contributions: R.F.L. and D.M. designed research; R.F.L., C.H., W.G., and D.L. performed research; R.F.L., C.H., W.G., and D.M. analyzed data; R.F.L. and D.M. wrote the paper.

This research was supported by NIH Grants DC6909 and DC8362. We thank Dr. Ruth Anne Eatock for comments on the manuscript.

The authors declare no financial conflicts of interest.

Correspondence should be addressed to Richard F. Lewis, 243 Charles Street, Boston, MA 02114. E-mail: Richard_lewis@meei.harvard.edu.

DOI:10.1523/JNEUROSCI.0112-13.2013

Copyright $\odot 2013$ the authors $\quad 0270-6474 / 13 / 339530-06 \$ 15.00 / 0$
}

gular velocity in a reasonably physiologic manner. In the maculae of the otolith organs (which sense linear acceleration of the head and its orientation relative to gravity), however, the polarities of the hair cells are not aligned (Spoendlin, 1966), and hence electrical stimulation of otolith afferents (Suzuki et al., 1969) does not approximate the complex modulation in afferent activity that occurs with changes in gravito-inertial force (GIF). For these reasons, work has focused on the development of a semicircular canal prosthesis that senses angular head velocity and provides this information to the brain by stimulating canal ampullary nerves with implanted electrodes (Lewis et al., 2010; Dai et al., 2011), and it is clear that this approach can generate a VOR response that reduces retinal image motion during head rotation.

While the VOR is easily quantified, patients with vestibular dysfunction also describe prominent perceptual and postural symptoms, and to evaluate the potential clinical utility of a canal prosthesis it is therefore necessary to investigate the more complex, integrative behavioral parameters related to perception and posture. There is considerable evidence that the brain uses angular velocity signals provided by the semicircular canals to estimate head orientation relative to gravity (Glasauer, 1992; Angelaki et al., 1999; Merfeld et al., 1999). Canal rotational cues provided by a prosthesis could potentially influence the perception of head orientation through this mechanism. In addition, body orientation relative to gravity appears to be calculated by combining vestibular (head relative to gravity) and neck proprioceptive (head relative to body) cues (Stapley et al., 2006). Rotational information provided by a canal prosthesis, therefore, also has the potential to improve postural control through its effect on the 
brain's estimate of head orientation. In this study, we directly tested the hypothesis that electrical stimulation of canal afferents alters the brain's estimate of head orientation, and found that posterior canal stimulation in rhesus monkeys produced aberrant percepts of head tilt, results that support the potential utility of a canal prosthesis to improve perception of spatial orientation and postural control in patients suffering from vestibular deficits.

\section{Materials and Methods}

Overview. Since canal afferents encode angular head velocity, electrical stimulation of the right posterior canal should provide the brain with information about the head's angular velocity that is qualitatively similar to the canal input produced by an actual head rotation about the sensitive axis of the right posterior canal (except for the absence of modulation in the paired left anterior canal and the difference in afferent dynamics). Activation of the right posterior canal produces both roll and pitch angular velocity cues, with the roll component directed toward the right and the pitch component directed upward. For the purposes of this experiment, we have focused on the roll component and investigated whether a canal cue that encodes roll velocity toward the right results in an aberrant perception of head tilt toward the right-ear-down position, which would be evidenced by a leftward deviation of the subjective visual vertical (SVV) light bar such that it is aligned parallel to the misperceived direction of gravity.

The experiments were performed on two juvenile female rhesus monkeys that previously had been used in a study investigating tilt perception in the normal rhesus. Results from this study demonstrated that the monkeys oriented the light bar very close to the true earth-vertical during static and dynamic roll tilts (Lewis et al., 2008). All methods were approved by the institutional animal care and use committee and were in accordance with U.S. Department of Agriculture guidelines.

Instrumentation. Three surgical procedures were performed under general anesthesia. A head bolt was implanted in the skull and was pitched forward $18^{\circ}$ with respect to the stereotactic zero so that when the head was immobilized in the monkey chair the lateral canals were close to alignment with the earth-horizontal, and the vertical canals close to alignment with the earth-vertical. A headcap affixed to the bolt was used to house the connectors for the eye coils and the ear electrode. Eye coils were implanted in the frontal plane of each eye, and a coil was implanted in the sagittal plane of the right eye using standard methods. The frontal eye coils were used to measure horizontal and vertical eye position, and the sagittal coil was used to measure torsional position. Finally, a stimulating electrode was inserted into the right posterior canal through an opening near the ampulla using the methods we previously described for squirrel monkey lateral canal stimulation (Merfeld et al., 2007; Lewis et al., 2010). The electrode was made from platinum wire (diameter 150 $\mu \mathrm{m})$ with $\sim 150 \mu \mathrm{m}$ of Teflon insulation stripped from the tip. The return path for the stimulation was an electrode inserted into the ipsilateral temporalis muscle. The position of the stimulating electrode was adjusted with a micromanipulator until maximal vertical and torsional eye movements were produced in the plane of the instrumented canal (e.g., downward and clockwise while facing the monkey) by short pulse trains without horizontal eye movements or twitching of the facial muscles. The monkeys displayed no behavioral evidence of vestibular damage (e.g., nystagmus, imbalance) after they had recovered from the electrode surgery.

Subjective visual vertical training and testing. These methods were exactly as described in detail in our prior publication on rhesus monkey tilt psychophysics (Lewis et al., 2008). Briefly, the monkey sat in the primate chair and rotated a small steering wheel that rotated in the roll plane the orientation of a light bar presented on an LCD panel, which was viewed through a circular aperture. No visual orientation cues were available unless an earth-vertical reference line was provided on the LCD display for training purposes. This reference line was used to train the monkeys to rotate the light bar near the earth-vertical, and once they performed this task accurately the reference bar was removed and the monkeys were trained on the SVV task while upright and while tilted in the roll plane. During testing, the monkeys were upright and stationary, and no visual orientation cues were present. The room was completely dark during SVV testing except for the light emitted by the LCD panel. As previously described, during testing no constraints were imposed on the animals' SVV responses, and we used a small tolerance window and timing criteria to determine when the animal had rotated the light bar to its desired orientation. The monkeys were rewarded for all responses during testing, and no information was available to them regarding the accuracy of their SVV responses during testing. For each test, we initially recorded SVV responses for $15 \mathrm{~s}$ before electrical stimulation of the posterior canal was initiated, then for $60 \mathrm{~s}$ during tonic stimulation, and then for $15 \mathrm{~s}$ after stimulation was stopped. A maximum of eight SVV tests were obtained on a given day, and a minimum of six tests were obtained in each monkey at each of the four stimulation frequencies.

Electrical stimulation. Like our prior studies (Merfeld et al., 2007; Lewis et al., 2010), electrical stimulation consisted of charge-balanced biphasic current pulses with cathodic, off, and anodic phases, each lasting $200 \mu \mathrm{s}$. We chose the current amplitude of the pulses used during the experiments by slowly increasing the current while recording the eye movements and viewing the monkey's face with an infrared camera. The current was fixed at an amplitude that produced large downward and clockwise torsional eye movements (e.g., in the plane of the stimulated right posterior canal) without horizontal eye movements or facial twitching. For monkey M1, the current amplitude used for the experiments was $90 \mu \mathrm{A}$, and for $\mathrm{M} 2$ it was $135 \mu \mathrm{A}$. The electrical stimulation provided to the monkeys before the SVV experiment was limited to the electrode characterization in the dark, as described above; neither animal received chronic prosthetic stimulation or stimulation in the light before these experiments were performed.

During experiments the rate of electrical stimulation ramped up from zero over $0.1 \mathrm{~s}$, was maintained at a tonic rate for $60 \mathrm{~s}$, and then ramped back down to zero over $0.1 \mathrm{~s}$. Four tonic stimulation rates were used: 60 , 100,150 , and 200 pulses per second (pps). While these stimulation rates do not define a precise angular head velocity, we can calculate an approximate rotational velocity based on known afferent physiology. In rhesus monkeys, irregular afferents have an average tonic rate of $87 \mathrm{spikes} / \mathrm{s}$ and an average sensitivity of $1.13 \mathrm{spikes} / \mathrm{s} / \%$, while regular afferents have an average tonic rate of 99 spikes/s and an average sensitivity of 0.47 spikes/ $\mathrm{s} / \%$ s (Cullen and Minor, 2002). If we assume that an equal number of irregular and regular afferents are entrained at the stimulation rate provided by the electrode, then the mean change in discharge rate provided by 200 pps stimulation would be 107 spikes/s and the mean afferent sensitivity would be $0.8 \mathrm{spikes} / \mathrm{s} / \mathrm{deg} / \mathrm{s}$, so this rate of stimulation would correspond to an angular velocity about the right posterior canal of $\sim 124^{\circ}$ /s (e.g., 107 spikes/s and 0.8 spikes/s/deg/s).

Eye movement recording. Three-dimensional eye movements were recorded from the right eye, and two-dimensional eye movements were recorded from the left eye. We used a right-hand-rule sign convention, with positive responses being defined as leftward, downward, and counterclockwise (while viewing the monkey, e.g., top of the eye rotating toward the right ear). Horizontal and vertical eye movements were calibrated with a behavioral calibration that related voltage measured by the frontal coils with eye position during target fixation. Torsional coils were calibrated with a gimble using a fixed relationship between voltage and degrees $\left(5 \mathrm{~V}=20^{\circ}\right)$. Eye movements were filtered at $50 \mathrm{~Hz}$ and sampled at a rate of $200 \mathrm{~Hz}$ for off-line analysis, and then digitally differentiated to yield an eye velocity time series.

Eye movements were recorded in the following three different conditions: (1) while the monkey performed the SVV task in an otherwise dark room, with the head upright; (2) in complete darkness, with the head upright; and (3) in complete darkness, with the head positioned statically so that the rotational axis of the stimulated right posterior canal was aligned with gravity. The purpose of the third test was to record the eye movements produced by posterior canal stimulation, while any potentially confounding interactions between the rotational cue produced by posterior canal stimulation and the gravitational cues sensed by the otoliths were removed. 


\section{Results}

Figure 1 illustrates the eye movements produced by right posterior canal stimulation at 200 pps in monkey M1 in the dark with the head positioned such that the rotational axis of the right posterior canal was aligned with gravity. A brisk vertical-torsional nystagmus was induced with slow phases directed downward and clockwise. The pattern of nystagmus produced by electrical stimulation therefore approximated the response that would occur if the head was actually rotated about the sensitive axis of the right posterior canal. The peak vertical eye velocity was $38 \%$, and the peak torsional velocity was $29 \%$ s. These values are close to those predicted from the $124 \%$ s angular velocity about the right posterior canal axis that we estimated above. In particular, the roll and pitch component of this angular velocity are cosine $(45) \times 124=88 \%$ s. In rhesus monkeys, the pitch VOR gain is $\sim 0.57$ and the roll VOR gain is $\sim 0.38$ (Angelaki and Hess, 1996), but since only one of the canal pairs is being modulated the predicted gain in this experiment is one-half of the normal gains (Paige, 1983), so gains are estimated to be 0.29 (pitch) and 0.19 (roll). The predicted eye velocities produced by 200 pps stimulation are therefore $0.29 \times 88=26 \%$ s (vertical) and $0.19 \times 88=17 \%$ s (torsional), which are approximately comparable to those we recorded.

Other than the nystagmus, no changes in torsional or vertical eye position were observed during this or other eye movement trials. In particular, no conjugate or disconjugate changes in the vertical position of the eyes were recorded nor was there any static change in the torsional position of the right eye. As evident in this eye movement trace, the monkey invariably made a horizontal saccade toward the stimulated right ear with a latency of $\sim 160$ $\mathrm{ms}$, but following that saccade minimal horizontal nystagmus was recorded.

When the monkeys were upright and performed the SVV task, their responses before stimulation onset were very close to the earth-vertical, but right posterior canal stimulation produced a nearly immediate deviation of the SVV response toward the left ear, consistent with a misestimate of head tilt toward the rightear-down position. Figure 2 shows sample SVV responses for both monkeys, and Figure 3 shows the mean and SE SVV response for both monkeys with each of the four stimulation rates. For both monkeys, the size of the SVV deviation scaled approximately linearly with the stimulation rate (Pearson correlation: M1, $r=0.73, p<0.001 ;$ M2, $r=0.76, p<0.001$ ), with no evidence of saturation over the stimulation range we used (Fig. 4 ). The peak torsional deviation of the right eye during stimulation is also shown in Figure 4 for trials in the dark and trials while the SVV task was performed. The peak torsional deviation of the eye was always smaller than the mean deviation of the SVV response ( $t$ test: M1 SVV vs torsion in dark, $p<0.001,41 \mathrm{df}$; SVV vs torsion during SVV, $p<0.001,40 \mathrm{df} ; \mathrm{M} 2 \mathrm{SVV}$ vs torsion in dark, $p=0.001,38 \mathrm{df}$; SVV vs torsion during SVV, $p<0.001,42 \mathrm{df}$ ),
Right eye
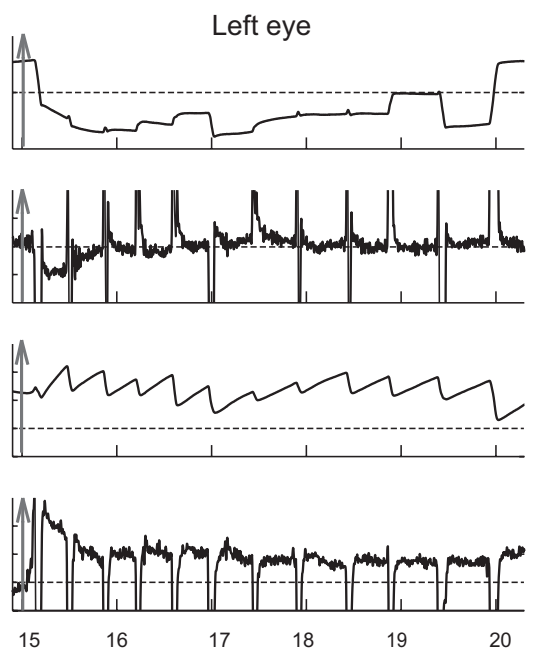

Time (s)
M1

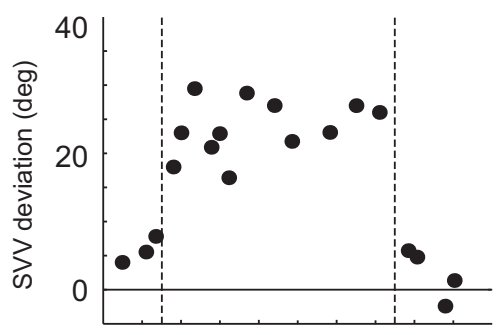

M2

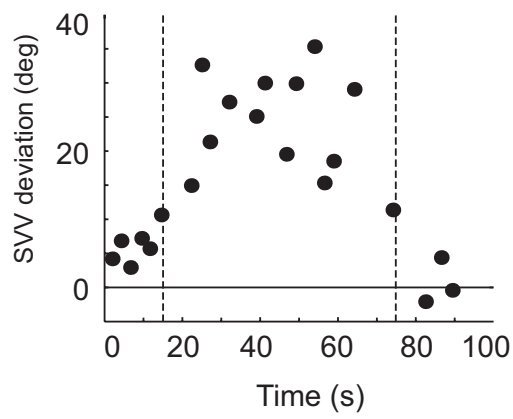

Figure 2. Sample trials showing the effect of right posterior canal stimulation on SWV responses with the head upright. The vertical lines show the onset and offset of stimulation. Stimulation rates were 150 pps in M1 and 200 pps in M2. Positive values represent angular deviation of the light bar toward the monkey's left ear (counterclockwise from the monkey's perspective). 

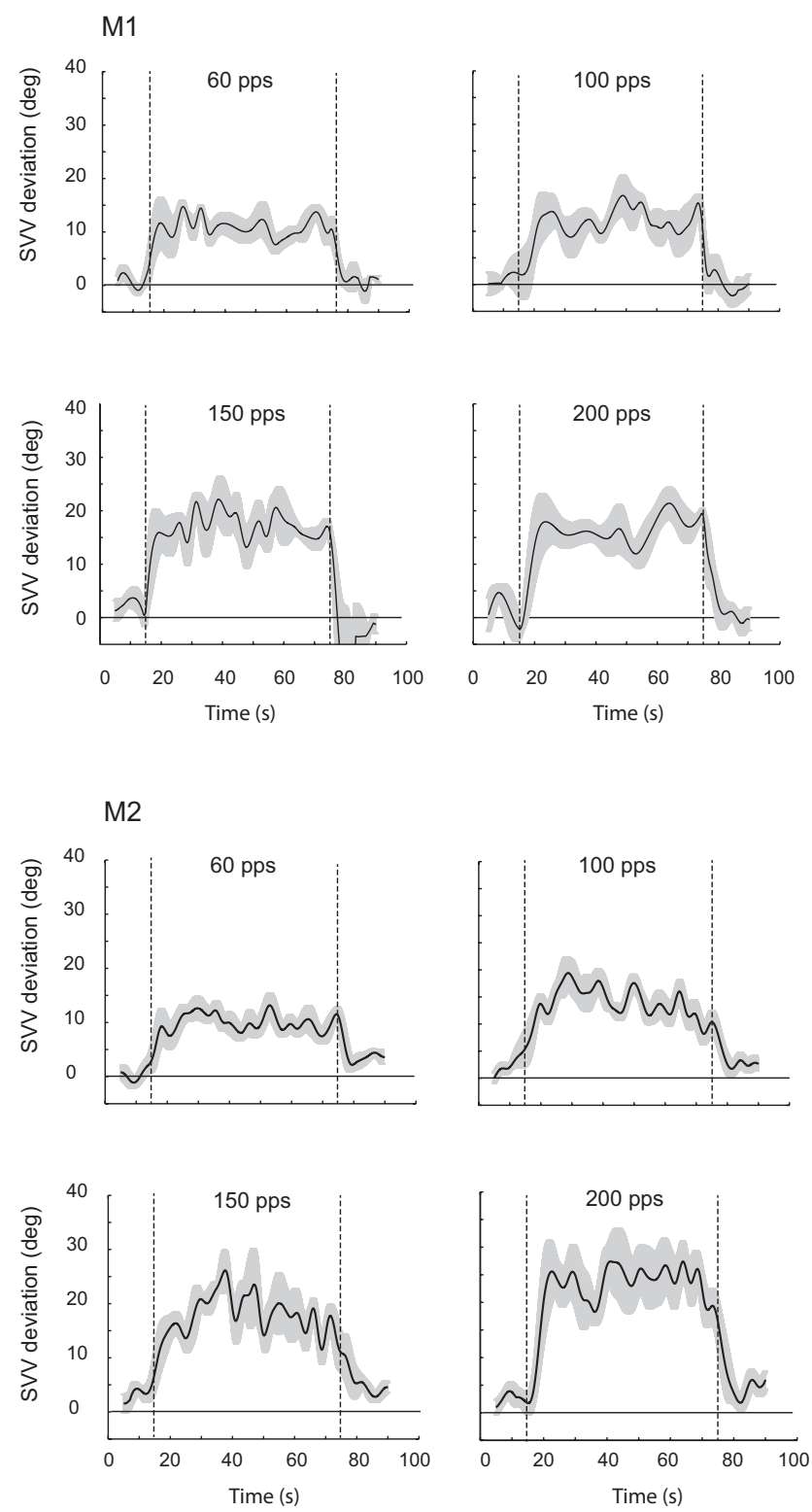

Figure 3. Mean and SEM for all stimulation trials in M1 and M2. Vertical lines indicate stimulation onset and offset. A spline interpolation between individual SVV responses was used to generate a continuous SVV variable for each trial, and the number of trials producing these plots for $\mathrm{M} 1$ are $6(60,100,200$ pps) and 7 (150 pps); and for M2 are 7 (60 pps), 9 (100 pps, 150 pps), and 6 (200 pps).

and the torsional deviation did not increase linearly with stimulation rate (Pearson correlation: $\mathrm{M} 1$ torsion in dark, $p=0.24$; torsion during SVV, $p=0.35$; M2 torsion in dark, $p=0.57$; torsion during SVV, $p=0.40$ ) like the mean SVV deviation. These findings demonstrate that the SVV changes were not purely visual in origin and did not simply reflect a shift in the torsional position of the eyes.

\section{Discussion}

Our results demonstrate that the roll angular velocity cues produced by activation of one posterior canal result in a misperception of head orientation in the roll plane. Before considering these findings further, we must evaluate what vestibular endorgans were activated by the stimulation. More specifically, it is critical to consider whether otolith afferents could have been
M1
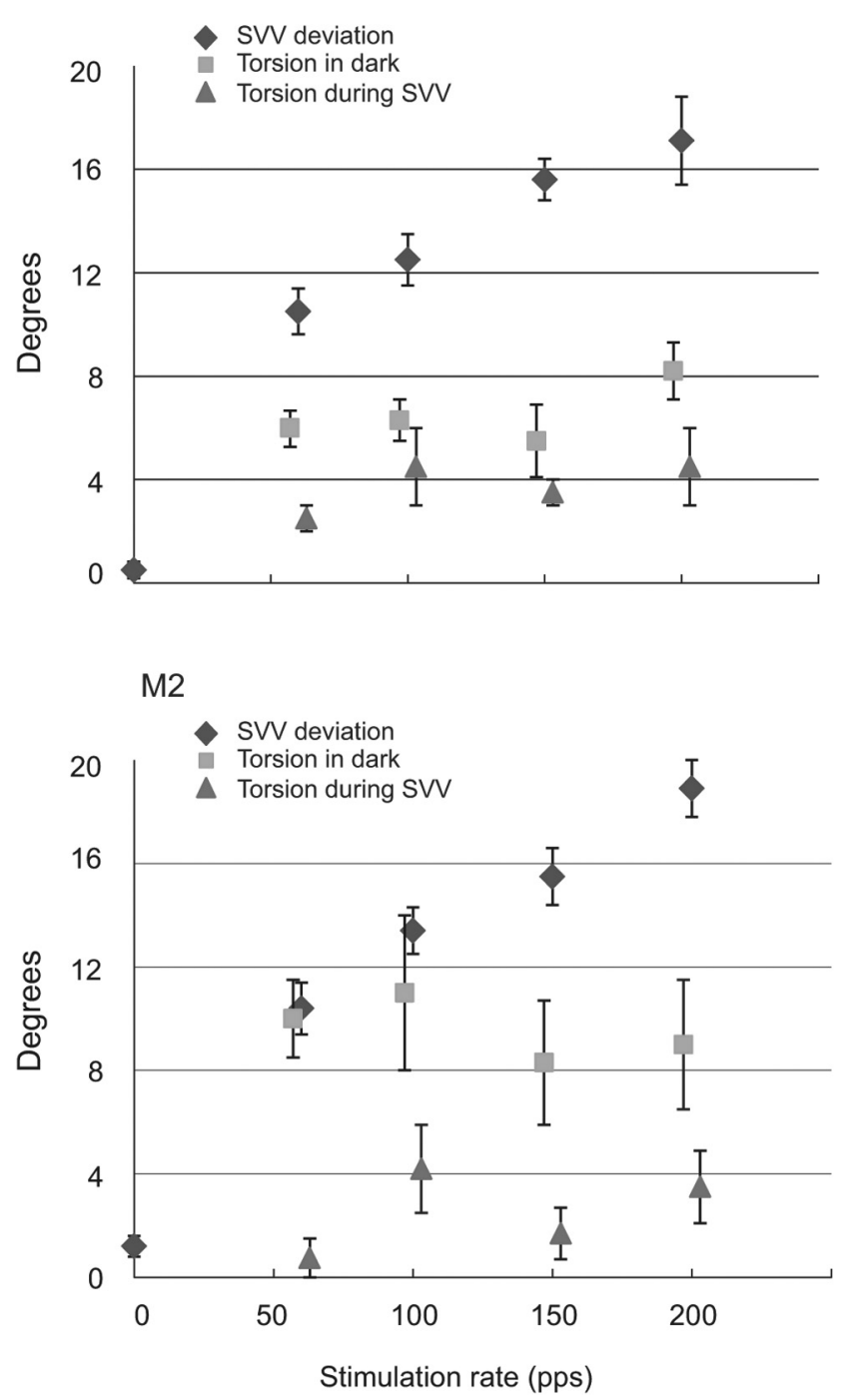

Figure 4. Magnitude of the mean and SEM for the average SVV deviation and for the maximal torsional deviation of the right eye produced by posterior canal stimulation. Values were calculated as the response during stimulation minus the response before stimulation, and are plotted against the frequency of stimulation. SVV results with a stimulation rate of zero were calculated from responses in the $15 \mathrm{~s}$ before stimulation onset.

activated, and if they were activated, what behavioral response would be predicted.

Since eye movements were consistent with isolated activation of the implanted posterior canal, the majority of stimulated vestibular afferents must be those innervating the right posterior canal. Significant current spread to other vestibular end-organs seems unlikely. To minimize current spread, we limited current levels to $135 \mu \mathrm{A}$ and used high-frequency pulsatile stimulation (Rubinstein, 1991). Consistent with this supposition, we found during implantation of the electrode that our ability to induce eye movements was highly dependent on the precise location of the electrode tip; movements as small as $100 \mu \mathrm{m}$ had a prominent effect on our ability to elicit the vertical and torsional eye movements reflective of posterior canal activation.

With the head upright, the utricles are nearly aligned with the earth-horizontal and the saccules are close to the earth-vertical (Spoendlin, 1966). When the head tilts in roll by relatively small angles, therefore, changes in shear force applied to the utricles are 
much larger than changes in force applied to the saccules. Since the utricles are the primary sensors of head orientation in this situation (Rader et al., 2009), we must address the possibility that utricular afferents could have been activated by the stimulation applied in the posterior canal. Eye movements produced by electrical stimulation of the utricular nerve consist of static contralateral torsional deviation and static disconjugate vertical deviation of the eyes, with the ipsilateral eye shifting upward and the contralateral eye shifting downward (Suzuki et al., 1969; Curthoys, 1987). With right-sided stimulation, therefore, utricular nerve activation should produce clockwise (negative) static torsional deviation of the eyes, upward (negative) deviation of the right eye, and downward (positive) deviation of the left eye. None of these movements were observed, providing strong evidence that utricular afferents were not significantly activated. Eye movements produced by saccular nerve stimulation are relatively weak and consist of upward or upward and torsional deviations of one or both eyes (Curthoys, 1987; Isu et al., 2000). As noted above, static shifts in the vertical or torsional position of the eyes were not observed during posterior canal stimulation, implying that significant electrical stimulation of saccular afferents was unlikely.

If otolith afferents were stimulated to a small degree that was not reflected in eye movement responses, what would be the effect on perceived head orientation? There is no animal literature regarding this issue, so we must consider the closest analogous type of stimulation in humans, galvanic vestibular stimulation (GVS). The effects of our electrical stimulation are analogous to cathodic GVS in that a depolarizing effect on nerve afferents is expected (Goldberg et al., 1984). Since the hair cells in the otolith maculae are not aligned but reverse orientation at the striola (Spoendlin, 1966), if electrical stimulation depolarized the utricular afferents, it would not produce the same pattern of modulation in utricular afferents produced by a head tilt. During roll tilt of the head toward the right, the firing rate of utricular afferents innervating the pars lateralis in the right ear decreases, and the firing rate of those afferents innervating the pars medialis on the right increases (Fernandez et al., 1972), and it is the difference in modulation between these two regions of the macula (which is reversed in the contralateral ear) that is thought to encode the change in head orientation. If the utricular nerve (or macula) is activated electrically on the right, however, firing increases in afferents innervating both the pars lateralis and medialis, and this pattern of afferent modulation should not encode head tilt but rather should nearly cancel (Fitzpatrick and Day, 2004). Since the pars lateralis is slightly larger than the pars medialis in humans (Tribukait and Rosenhall, 2001), stimulation of the right utricular nerve is predicted to induce a weak illusion of leftward head tilt, behavior that has been documented in several human GVS studies (Zink et al., 1997, 1998) and is in the opposite direction of our results. In rhesus monkeys, the relative sizes of the pars lateralis and medialis are unknown so it is unclear whether stimulation of utricular afferents would induce an illusion of tilt toward or away from the stimulated ear. It is important to emphasize, however, that the net "tilt" effect produced by electrical stimulation of utricular afferents depends on the extent of asymmetry between the pars lateralis and medialis, and since these regions are very similar in size in the human and nonhuman primates (Fernandez et al., 1972) where it has been measured, any tilt response produced by electrical stimulation in rhesus monkeys, if it occurred, is almost certainly very small. In sum, a reasonable conclusion is that posterior canal activation produced all, or the vast majority, of the tilt response we observed in these experiments, since our eye movement analysis showed no evidence of otolith stimulation, and even if a small amount of otolith stimulation did occur, its effect on perceived tilt is expected to be very small and possibly in the direction opposite to the response recorded in the monkeys.

The effects of torsional eye position on the perceived orientation of the SVV light bar remain controversial (Wade and Curthoys, 1997; Mast, 2000). While some studies have shown a close correlation between the orientation of the light bar and torsional eye position (Pavlou et al., 2003), our results demonstrated that the SVV deviation was significantly larger than the torsional eye deviation. The SVV shift also increased approximately linearly with increasing stimulation rate, while the torsional deviation did not correlate with the rate of stimulation. While changes in torsional eye position may have contributed to the SVV deviation we observed, they were clearly of insufficient magnitude to be the predominant mechanism responsible for the SVV response, and therefore posterior canal stimulation must have affected the brain's estimate of head orientation relative to gravity.

The SVV deviation produced by electrical stimulation of the posterior canal cannot be compared with tilt percepts generated by head rotations that activate the posterior canal without modulating otolith cues, since this experiment has never been performed. The closest approximation in the literature is optokinetic stimulation, which has also been shown to affect percepts of head orientation when presented in the roll plane (Dichgans et al., 1972). As described above, we estimate that 200 pps stimulation applied to one posterior canal approximated a roll angular velocity of $\sim 88^{\circ} / \mathrm{s}$, and this resulted in a mean SVV deviation in the two monkeys of $18^{\circ}$. This result is close to the $15^{\circ}$ shift in perceived upright produced by a roll optokinetic stimulus moving at a similar velocity (Dichgans et al., 1972), suggesting that angular velocity signals in the roll plane provided by canal or optokinetic stimulation have similar effects on perceived head orientation.

An accurate estimate of head orientation relative to gravity is critically important for both spatial perception (Barra et al., 2010) and postural control (Peterka, 2002; Stapley et al., 2006). While the brain's estimate of head orientation clearly depends on a synthesis of several sensory modalities including vision and proprioception, studies in vestibular-deficient patients (Valko et al., 2012) and animals (Lewis et al., 2011) clearly demonstrate that vestibular information also contributes to this calculation. The mechanism by which vestibular signals help estimate head orientation is complicated by the fact that the otolith organs sense the vector sum of gravity and the inertial force due to linear acceleration (GIF), and hence do not provide an unambiguous measure of head orientation relative to gravity. The angular velocity signals and GIF signals are not independent, however, since the canals and otolith organs are mechanically coupled in the temporal bone. It has therefore been hypothesized (and supported by a number of studies: Angelaki et al., 1999; Merfeld et al., 2005) that head orientation relative to gravity is estimated by the brain by integrating the vector cross-product of the estimated angular head velocity (derived from canal inputs) and the direction of gravity (derived from otolith inputs). Our results support this putative mechanism, since we demonstrated that modulating activity in canal afferents without any concomitant changes in GIF shifted the perceived orientation of the head. Prior work has shown that electrical stimulation of canal afferents can produced VOR responses that reduces retinal image motion (Dai et al., 2011), undergo adaptation (Lewis et al., 2010), and interact with gravitational cues (Lewis et al., 2012). Our work, in contrast, 
provides the first evidence that a canal prosthesis can modify the brain's estimate of head orientation relative to gravity. This finding has significant implications, since it suggests that a canal prosthesis could potentially improve both percepts of head orientation and postural control in patients suffering from a reduction in peripheral vestibular function.

\section{References}

Angelaki DE, Hess BJ (1996) Adaptation of primate vestibuloocular reflex to altered peripheral vestibular inputs. II. Spatiotemporal properties of the adapted slow-phase eye velocity. J Neurophysiol 76:2954-2971. Medline

Angelaki DE, McHenry MQ, Dickman JD, Newlands SD, Hess BJ (1999) Computation of inertial motion: neural strategies to resolve ambiguous otolith information. J Neurosci 19:316-327. Medline

Barra J, Marquer A, Joassin R, Reymond C, Metge L, Chauvineau V, Pérennou D (2010) Humans use internal models to construct and update a sense of verticality. Brain 133:3552-3563. CrossRef Medline

Cullen KE, Minor LB (2002) Semicircular canal afferents similarly encode active and passive head-on-body rotations: implications for the role of vestibular efference. J Neurosci 22:RC226. Medline

Curthoys IS (1987) Eye movements produced by utricular and saccular stimulation. Aviat Space Environ Med 58:192-197.

Dai C, Fridman GY, Davidovics NS, Chiang B, Ahn JH, Della Santina CC (2011) Restoration of 3D vestibular sensation in rhesus monkeys using a multichannel vestibular prosthesis. Hear Res 281:74-83. CrossRef Medline

Dichgans J, Held R, Young LR, Brandt T (1972) Moving visual scenes influence the apparent direction of gravity. Science 178:1217-1219. CrossRef Medline

Diener HC, Dichgans J (1988) On the role of vestibular, visual, and somatosensory information for dynamic postural control in humans. Prog Brain Res 76:253-262. CrossRef Medline

Fernandez C, Goldberg JM, Abend WK (1972) Response to static tilts of peripheral neurons innervating otolith organs of the squirrel monkey. J Neurophysiol 35:978-987. Medline

Fitzpatrick RC, Day BL (2004) Probing the human vestibular system with galvanic stimulation. J Appl Physiol 96:2301-2316. CrossRef Medline

Glasauer S (1992) Interaction of semicircular canals and otoliths in the processing structure of the subjective zenith. Ann N Y Acad Sci 656:847-849. CrossRef Medline

Goldberg JM, Smith CE, Fernández C (1984) Relation between discharge regularity and responses to externally applied galvanic currents in vestibular nerve afferents of the squirrel monkey. J Neurophysiol 51:1236-1256. Medline

Isu N, Graf W, Sato H, Kushiro K, Zakir M, Imagawa M, Uchino Y (2000) Sacculo-ocular reflex connectivity in cats. Exp Brain Res 131:262-268. CrossRef Medline

Lewis RF (1996) Vertigo: some uncommon causes of a common problem. Semin Neurol 16:55-62. CrossRef Medline

Lewis RF, Haburcakova C, Merfeld DM (2008) Roll tilt psychophysics in rhesus monkeys during vestibular and visual stimulation. J Neurophysiol 100:140-153. CrossRef Medline

Lewis RF, Haburcakova C, Gong W, Makary C, Merfeld DM (2010) Vestibuloocular reflex adaptation investigated with chronic motionmodulated electrical stimulation of semicircular canal afferents. J Neurophysiol 103:1066-1079. CrossRef Medline

Lewis RF, Haburcakova C, Gong W, Wall C, Thompson L, Karmali F, Lee D, Merfeld DM (2011) Responses evoked by a vestibular implant providing chronic stimulation. ARO Abstr 262:2011.

Lewis RF, Haburcakova C, Gong W, Karmali F, Merfeld DM (2012) Spatial and temporal properties of eye movements produced by electrical stimu- lation of semicircular canal afferents. J Neurophysiol 108:1511-1520. CrossRef Medline

Mast FW (2000) Does the world rock when the eyes roll? Swiss J Psychol 59:89-101. CrossRef

Merfeld D (2012) Spatial orientation and the vestibular system. In: Sensation and perception, Ed 3 (Carroll S, ed), pp 328-361. Sunderland, MA: Sinauer Associates.

Merfeld DM, Lewis RF (2012) Replacing semicircular canal function with a vestibular implant. Curr Opin Otolaryngol Head Neck Surg 20:386-392. CrossRef Medline

Merfeld DM, Zupan L, Peterka RJ (1999) Humans use internal models to estimate gravity and linear acceleration. Nature 398:615-618. CrossRef Medline

Merfeld DM, Park S, Gianna-Poulin C, Black FO, Wood S (2005) Vestibular perception and action employ qualitatively different mechanisms. II. VOR and perceptual responses during combined tilt and translation. J Neurophysiol 94:199-205. CrossRef Medline

Merfeld DM, Haburcakova C, Gong W, Lewis RF (2007) Chronic vestibuloocular reflexes evoked by a vestibular prosthesis. IEEE Trans Biomed Eng 54:1005-1015. CrossRef Medline

Minor LB (1998) Gentamicin-induced bilateral vestibular hypofunction. JAMA 2789:541-544. CrossRef Medline

Paige GD (1983) Vestibuloocular reflex and its interactions with visual following mechanisms in the squirrel monkey. II. Response characteristics and plasticity following unilateral inactivation of horizontal canal. J Neurophysiol 49:152-168. Medline

Pavlou M, Wijnberg N, Faldon ME, Bronstein AM (2003) Effect of semicircular canal stimulation on the perception of the visual vertical. J Neurophysiol 90:622-630. CrossRef Medline

Peterka RJ (2002) Sensorimotor integration in human postural control. J Neurophysiol 88:1097-1118. Medline

Rader AA, Oman CM, Merfeld DM (2009) Motion perception during variableradius swing motion in darkness. J Neurophysiol 102:2232-2244. CrossRef Medline

Rubinstein JT (1991) Analytical theory for extracellular electrical stimulation of nerves with focal electrodes. II. Passive myelinated axon. Biophys J 60:538-555. Medline

Spoendlin H (1966) The ultrastructure of the vestibular sense organs. In: The vestibular system and its diseases (Wolfson RJ, ed). Philadelphia: University of Pennsylvania.

Stapley PJ, Ting LH, Kuifu C, Everaert DG, Macpherson JM (2006) Bilateral vestibular loss leads to active destabilization of balance during voluntary head turns in the standing cat. J Neurophysiol 95:3783-3797. CrossRef Medline

Suzuki JI, Tokumasu K, Goto K (1969) Eye movements from single utricular nerve stimulation in the cat. Acta Otolaryngol 68:350-362. CrossRef Medline

Tribukait A, Rosenhall U (2001) Directional sensitivity of the human macula utriculi based on morphological characteristics. Audiol Neurootol 6:98-107. CrossRef Medline

Valko Y, Lewis RF, Priesol AJ, Merfeld DM (2012) Vestibular labyrinth contributions to human whole-body motion discrimination. J Neurosci 32: 13537-13542. CrossRef Medline

Wade SW, Curthoys IS (1997) The effect of ocular torsional position on perception of the roll-tilt of visual stimuli. Vision Res 37:1071-1078. CrossRef Medline

Zink R, Steddin S, Weiss A, Brandt T, Dieterich M (1997) Galvanic vestibular stimulation in humans: effects on otolith function in roll. Neurosci Lett 232:171-174. CrossRef Medline

Zink R, Bucher SF, Weiss A, Brandt T, Dieterich M (1998) Effects of galvanic vestibular stimulation on otolith and semicircular canal eye movements and perceived vertical. Electroencephalogr Clin Neurophysiol 107:200205. CrossRef Medline 